\title{
Using DTMon to Monitor Transient Flow Traffic
}

\author{
Hadi Arbabi and Michele C. Weigle \\ Department of Computer Science \\ Old Dominion University \\ Norfolk, VA 23529 USA \\ \{marbabi, mweigle\}@cs.odu.edu
}

\begin{abstract}
We evaluate the performance of the DTMon dynamic traffic monitoring system to measure travel times and speeds in transient flow traffic caused by non-recurring congestion. DTMon uses vehicular networks and roadside infrastructure to collect data from passing vehicles. We show DTMon's ability to gather high-quality real-time traffic data such as travel time and speed. These metrics can be used to detect transitions in traffic flow (e.g., caused by congestion) especially where accurate flow rate information is not available. We evaluate the accuracy and latency of DTMon in providing traffic measurements using two different methods of message delivery. We show the advantages of using dynamically-defined measurement points for monitoring transient flow traffic. We compare DTMon with currently in-use probe-based systems (e.g., AVL) and fixed-point sensors and detectors (e.g., ILD).
\end{abstract}

\section{INTRODUCTION}

Real-time monitoring of traffic is an important function of traffic management centers (TMCs). To achieve accurate monitoring requires real-time observation of vehicular mobility in traffic. Metrics that TMCs collect include flow rate and time mean speed (TMS), the average speed of vehicles passing a point on the roadway. These two metrics are traditionally collected by sensors and detectors at fixed points on the road. Other metrics, such as travel time and space mean speed (SMS), the average speed based on the travel time in a particular segment, rely on information gathered from the same vehicles passing more than one point on the road. These metrics cannot be directly measured by point sensors and currently must be estimated. In free-flow, or uncongested, traffic, this estimation is usually sufficient. But, in transient flow traffic, for instance during periods of congestion, these estimates are often highly inaccurate. Unfortunately, it is during such periods of congestion that accurate travel times and SMS measurements are most needed.

In previous work [1], we have shown how dynamic traffic monitoring using vehicular networks (DTMon) [2] can be used to gather high-quality travel time and SMS data in free-flow traffic. In this paper, we analyze and evaluate the ability of DTMon to gather accurate information in transient flow conditions as well. DTMon consists of roadside infrastructure we call task organizers (TOs) and vehicles equipped with communications devices. TOs use Dedicated Short Range Communication (DSRC) [3] to communicate with passing vehicles and assign them measurement tasks. Vehicles report information back to the TO upon passing a specified measurement point, which we call a virtual strip (VS). The key of DTMon is that these VS can be positioned at any point along the roadway and changed dynamically by the TOs according to the traffic conditions.

In this paper, we evaluate the performance and ability of DTMon to gather real-time data on traffic with transient flow as a result of a non-recurring congestion. We evaluate DTMon under two methods of message delivery and compare the results with both point-based monitoring and probe-based monitoring technologies. We show that DTMon is capable of estimating high quality travel time and SMS data even in situations where a small percentage of vehicles are equipped for communications.

\section{BACKGROUND}

\section{A. Sensors and Detectors}

Sensors and detectors, such as inductive loop detectors (ILDs), video cameras, microwave radar sensors (MRS), and acoustic radars, are currently the most commonly-used technologies for traffic monitoring [4]. These devices must be placed at particular locations along the roadway. Thus, installation and maintenance have non-negligible costs. Although each of these types of devices has its own advantages and disadvantages, as a whole these point-based sensors and detectors can count the number of passing vehicles, and therefore, compute the traffic flow rate over certain time intervals. They can estimate each vehicle's speed, which allows them to estimate the TMS. Because they can count the number of passing vehicles, they can approximate the traffic density, the number of vehicles occupying a certain area.

Yet, the type of data gathered by point-based sensors is not sufficient. The only way to obtain an accurate picture of traffic on any particular road segment is to deploy many of these sensors along the road segment, which is not cost efficient or feasible (e.g., installment, synchronization, operation, and maintenance are issues). Identifying the same vehicle passing two points of a segment, needed for computing accurate travel times, is not possible with such sensors. In addition, these sensors and detectors cannot inform traveling vehicles with generated reports or up-to-date traffic conditions.

\section{B. Probe-Based Systems}

Recent developments in gathering segment-oriented traffic data (e.g., travel time, SMS) have been largely probe-based technologies, which allow individual vehicles to report statistics as they move through traffic. The most prominent probe-based technologies are automatic vehicle location (AVL) and wireless location tracking (WLT) systems $[4,5,6]$. AVL systems are typically deployed in freight vehicles to assist 
owners with tracking their shipments, and thus the data produced is proprietary and not widely shared. In addition, as freight trucks have different travel characteristics than most other vehicles, the collected data is not a highly accurate picture of the overall traffic. WLT systems track the presence of mobile phones in vehicles, but they typically rely on coarsegrained positioning (based on cell towers) that cannot reliably identify roadways. In addition, they count each phone as a different vehicle, even though a single vehicle may contain multiple phones, so the data collected is not yet high quality. Probe-based systems generally have issues due to sampling period and sample size. Vehicles periodically send updates on their position at specific intervals, so the time that a vehicle passed a particular point (and its speed) must be interpolated using samples taken at different times and locations. Having an accurate position and speed of vehicles requires a higher sampling rate, which would consume the radio channel bandwidth unnecessarily since an excessive amount of data will be transmitted. In our system, DTMon, vehicles can report data upon passing a particular monitoring point rather than just periodically. Another limitation to probe-based systems is that typically only a small subset of the vehicle population is equipped to gather statistics. This market penetration rate can greatly affect the quality of estimated metrics.

\section{Congestion}

There are two types of traffic congestion. Recurring congestion occurs regularly at the same locations on the roadway system at about the same time every day. Nonrecurring congestion occurs when a crash or other unusual event (such as a construction zone, merging traffic, or severe weather) causes a reduction in the traffic-carrying capacity. Detecting recurring congestion is not a critical monitoring process and requires a long-term overview (i.e., weekly, monthly, or annual) of a large archive of collected traffic data, estimated metrics, and structural features of the roads (e.g., capacity) in addition to extra data-mining to detect the recurrence of similar patterns, all of which can be done offline. Detecting and monitoring non-recurring congestion, on the other hand, is an important function of TMCs. Extended periods of non-recurring congestion may require immediate action, including notifying emergency personnel, highway patrols, or even changing traffic light timings on surface streets to handle the extra capacity from diverted highway vehicles.

\section{RELATED WORK}

Recent research on wireless traffic monitoring is focused on intelligently combining the best data from the traffic network to provide traffic information, directions and driver services. Inrix [5] uses combined collected data from various sources (i.e., sensors and detectors, AVL systems, and mobile nodes) to generate reports. Wireless technology and mobile nodes are the most recent available source for traffic monitoring. Cell phones are the main part of the architecture in the Mobile Millennium project [6]. PATH's Group-Enabled Mobility and Safety (GEMS) project [7] uses mobile nodes as clients of traffic reports. GEMS is based on AVL and WLT technologies with the use of Internet queries for delivering data to handheld devices. The challenge in AVL and WLT systems is to determine the minimum collection rate of positioning data required to achieve the desired level of travel time estimation accuracy. This minimum collection rate can vary in different traffic conditions and with various penetration rates and sample sizes [8,9]. CarTel [10] uses cell phones and cars as nodes in a dynamic sensor network. In this distributed mobile sensor computing system, software is used to collect, process, and visualize data from sensors located on mobile devices to a central portal. TrafficView [11] is a scalable traffic monitoring system for inter-vehicle communication considering road conditions, but it does not consider low market penetration rates or low traffic density. Kitani et al. [12] have proposed traffic information sharing using public buses traveling regular routes. This VANET-based technique is only useful in urban areas with good public transportation systems and only monitors those areas traveled by the transit system.

\section{OVERVIEW OF DTMON}

In this section, we provide an overview of the DTMon architecture. The main components are the roadside infrastructure, the equipment in each vehicle, and virtual strips.

\section{A. Task Organizer}

DTMon uses roadside infrastructure, called task organizers (TOs), to communicate with passing vehicles using a Dedicated Short Range Communications (DSRC) [3] device. The TO assigns measurement tasks, collects reports from the vehicles, organizes the received measurements, and informs vehicles about upcoming traffic conditions. The goal of the TO is to provide accurate measurement information to the TMC and to disseminate timely messages from the TMC to equipped vehicles. Multiple TOs may be deployed along a single roadway. We assume that all TOs in a region are connected to a central server at the TMC. This allows the TMC to provide aggregated information about the whole region.

\section{B. Vehicles}

Vehicles in DTMon are equipped with a Global Positioning System (GPS) device and a DSRC communications device. Vehicles record their speed, GPS position, and travel direction along with a timestamp. Each transmitted message from a vehicle contains a header that includes all of above information. Vehicles receive tasks from a TO, which can be triggered at a specific time, speed, or location. After completing a task, the vehicle produces a report that is either forwarded back to the TO or stored and carried to the next available TO.

\section{Virtual Strips}

A virtual strip (VS) defines a measurement point on the road. Two virtual strips can be used to create a virtual segment. Each strip can be represented either as a line or by a set of points, with one point for each lane (in the form of latitude, longitude, and altitude). We show an example of a highway with two TOs and five VS in Figure 1.

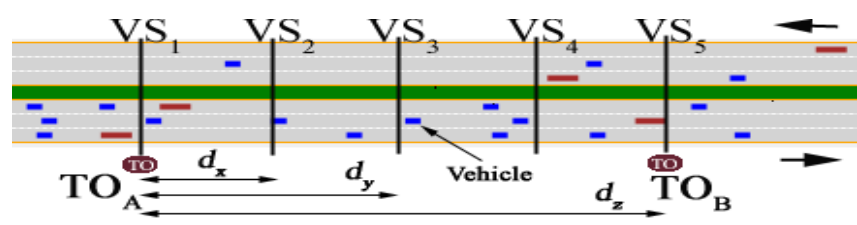

Figure 1. Five dynamically defined VS and two TOs. 


\section{Message Delivery}

In DTMon, measurement reports from vehicles are typically forwarded through the vehicular network to a TO. In this Regular Forwarding $(R F)$, a vehicle passing a VS will forward its report to the closest TO from the list of TOs defined in the task. The success of this forwarding depends upon several factors, including the transmission range of the DSRC devices (typically $300 \mathrm{~m}$ ), the inter-vehicle spacing between equipped vehicles, and the market penetration rate (PR). In many situations, especially in low density traffic or with low PR, messages that depend upon RF are not able to be reliably delivered to a TO. Therefore, methods other than RF must be considered to improve the amount of information that can be delivered in such traffic conditions.

In some situations, DTMon can use traffic traveling in the opposite direction for forwarding, which may reduce the intervehicle spacing of equipped vehicles and improve the amount of information received. In situations where there are multiple TOs on the roadway, the combination of RF and store-andcarry (SAC) can be used. In Regular Forwarding plus Storeand-Carry $(R F+S A C)$, a vehicle will attempt to forward the message to the closest TO using RF and will also store and carry the message to the next TO in order to ensure reception by a TO. Duplicate reports are detected by the central server at the TMC using the message generation time and location.

\section{DTMON IN TRANSIENT Flow TRAFFIC}

Because the coverage area of a single TO is limited and transient traffic conditions can cause periods of network disconnectivity, there is a need for multiple TOs in areas that are prone to experiencing non-recurring congestion events. When multiple TOs are used, message delay and latency may affect the quality of data that can be accurately gathered. In addition, it is important to carefully assign measurement points in transient traffic as the conditions can change quickly. We show how DTMon can allow for the dynamic creation of new measurement points (VS) and the movement of existing VS.

\section{A. Latency}

In DTMon, tasks can include the locations of multiple TOs. This adds the ability for messages to be stored and be dropped off at the next available TO during the travel (using RF+SAC). This will reduce the number of messages lost due to unsuccessful forwarding. In addition to covering a wider area, the use of multiple TOs can improve the message delivery mechanism. The delay between a vehicle passing a VS and delivering the report to a TO may be reduced in some instances. Furthermore, generated reports and traffic warnings by TOs can be spread faster and more reliably in the region.

Message delay is the delay from when a message was generated to when it was received by a TO. Since vehicles' timestamp all reports, the receiving TO can calculate the message delay for each message. When messages are delivered to multiple TOs in a region, there is additional processing delay associated with sending the message to the central TMC and having that data aggregated with previously received data. We use the term latency for the total time from when a message was generated to when it is ready to be used in analysis. In a single-TO system, the latency is the same as the message delay.
TMCs often aggregate data and use specific averaging intervals (e.g., $1 \mathrm{~min}, 5 \mathrm{~min}$ ) when reporting traffic conditions. Latency is important if it rises to higher than the averaging interval. In this case, some late-arriving data may not be included in the estimate.

Figure 2 shows two TOs and a single VS. The distances between the TOs and the VS are shown as $d_{i}$, and the free-flow travel time between the points is shown as $t_{i}$. In a bi-directional roadway, messages can be stored and carried from $\mathrm{VS}_{i}$ to $\mathrm{TO}_{1}$ by vehicles traveling in the opposite direction from the vehicle that generated the report. This may decrease the message delay when the travel time in the segment $\mathrm{TO}_{1} \mathrm{VS}_{i}$ in the opposite direction is lower than the travel time from $\mathrm{VS}_{i}$ to $\mathrm{TO}_{2}$.

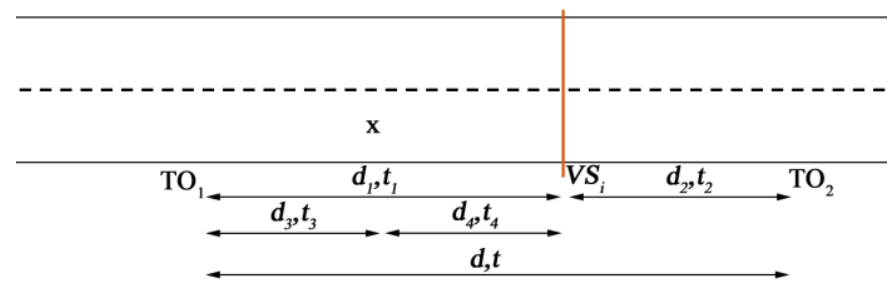

Figure 2. Two TOs, virtual strip $\mathrm{VS}_{\mathrm{i}}$, and actual travel times.

If there is some point (for instance, $x$ in Figure 2) past which a message cannot be forwarded and only one TO is used, there will be an unsuccessful message delivery. By adding an additional TO, this message can be stored and carried to $\mathrm{TO}_{2}$. The message delay of a message carried from $\mathrm{VS}_{i}$ to $\mathrm{TO}_{2}$ is $t_{2}$, which is the average travel time from $\mathrm{VS}_{i}$ to $\mathrm{TO}_{2}$. There is no need to forward the message back to $\mathrm{TO}_{1}$. If $\mathrm{VS}_{\mathrm{i}}$ is near $\mathrm{TO}_{2}$, the message can be forwarded to $\mathrm{TO}_{2}$ (using RF) with little delay (on the order of milliseconds) or be stored and carried to $\mathrm{TO}_{2}$ (using $\mathrm{RF}+\mathrm{SAC}$ ) with the average delay $t_{2}$, the travel time from $\mathrm{VS}_{i}$ to $\mathrm{TO}_{2}$.

In the case where a TMC can process reports delivered to either $\mathrm{TO}_{1}$ or $\mathrm{TO}_{2}$, messages can be forwarded in any direction or stored and carried to the closest TO. Here, the average message delay in a uni-directional roadway is:

$$
\begin{gathered}
\overline{\text { delay }}=\frac{\left(n_{f} \bar{t}_{f}+n_{c} \bar{t}_{c}\right)}{n} \\
\overline{\text { delay }}=w_{f} \bar{t}_{f}+w_{c} \bar{t}_{c}
\end{gathered}
$$

where

$$
\begin{aligned}
& n_{f}=\text { total number of distinct received forwarded messages } \\
& n_{c}=\text { total number of distinct received carried messages } \\
& n=\text { total number of distinct received messages } \\
& t_{f}=\text { forwarding delay } \approx 0.0 \\
& t_{c}=\text { carrying delay } \approx \text { average travel time } \\
& w_{f}=n_{f} / n, \text { percentage of messages that are forwarded } \\
& w_{c}=n_{c} / n, \text { percentage of messages that are carried }
\end{aligned}
$$

The average delay of message generated in segment $\mathrm{TO}_{1} \mathrm{TO}_{2}$ is $t / 2$, the average travel time from any VS inside the segment $\mathrm{TO}_{1} \mathrm{TO}_{2}$. The average delay for messages related to $\mathrm{VS}_{i}$ will be $w_{f} t_{l}+w_{c} t_{2}$. 
Note that the average travel time in each interval highly depends on the actual traffic conditions on the road. For example, a non-recurring congestion event between $\mathrm{VS}_{i}$ and $\mathrm{TO}_{2}$ will cause an increase in $t_{2}$. Therefore, all messages behind the congestion event that are stored to be carried will be delivered to $\mathrm{TO}_{2}$ with message delay higher than expected. In addition, if the density of equipped vehicles is high, most of the messages will be successfully forwarded, and $w_{c}$ will be small and the message delay will tend towards zero. In the case where all forwarded messages are dropped due to disconnectivity in the network, they will be carried, therefore $w_{f}$ will be small and the message delay will tend towards the average travel time.

If congestion occurs at a point $x$ in Figure 2, $t_{3}$ will increase which will cause an increase in the overall travel time $t_{l}$ (and a corresponding decrease in SMS) in any segment surrounding $x$ (e.g., $\mathrm{TO}_{1} \mathrm{VS}_{\mathrm{i}}$ ). This transition in travel time has no impact on message delay when RF is used, but it will impact message delay when $\mathrm{RF}+\mathrm{SAC}$ is used and $x$ is after $\mathrm{VS}_{\mathrm{i}} . \mathrm{TO}_{2}$ will receive the stored messages carried by vehicles with additional delay caused by the congestion.

\section{B. Dynamically Defined Virtual Strips}

The location of VS can be re-defined and modified by a TO at any time. A TO can divide the roadway into several virtual segments. The size of the segments can vary by relocating the VS. Also, additional VS can be defined inside any desired virtual segment. This allows the TO receive more traffic information by defining additional points of interest on the road without the cost of using additional TOs. Currently, achieving this would require the installment of numerous sensors and detectors along the road. In AVL and WLT systems, this would require a significant increase in the sampling rate to maintain the desired level of accuracy [9].

Congestion can produce a transition in traffic flow which cannot be detected by probe-based systems when the PR is not high. We show that the transition in the flow rate can produce a transition in travel time and SMS, which can be detected by DTMon. The travel time algorithms proposed by Sethi et al. [13] and Sermons [14] utilize both the travel time and average speed measures of the congested segment and the adjacent segment. DTMon using dynamically defined strips/segments can satisfy this data requirement. Two variables, travel time and speed, are compared to historical averages for each segment to infer if congestion occurs on this segment. It was found by Sethi that traffic measures for the congested segment were most useful for detecting congestion located in the downstream portion of the segment, while traffic measures for the next upstream segment worked well for detecting congestion occurring in the upstream or middle portion of the segment. The segment in the upstream portion that shows no significant change in traffic measures is inferred to be the endof-the-queue.

Identifying the end-of-the-queue is important for traffic engineers as this is the place where most secondary incidents occur. The primary incident is the cause of the congestion, but secondary incidents tend to occur at the point where the speed transition is the sharpest. Traffic engineers would like to be able to identify these locations so that approaching drivers can be warned in advance, potentially avoiding the secondary incidents.

\section{EVALUATION}

There are several factors that can affect the quality of data gathered by a VANET-based traffic monitoring system like DTMon. The first factor is the percentage of equipped vehicles, or the market penetration rate (PR). Second, we are concerned with the amount of information (i.e., location, speed, time) that is received from vehicles. Third, we must consider how quickly and in what manner the information is received. Methods that can collect more information from vehicles with less latency are preferred in up-to-date traffic monitoring.

The message reception rate (MRR) for a particular VS is the percentage of messages generated by equipped vehicles passing the strip that were received by the TO. The information reception rate (IRR) for a particular VS is the percentage of messages received by the TO out of all possible messages generated by vehicles passing the strip (i.e., as if all vehicles were equipped). The IRR indicates how well count information can be collected by the TOs under scenarios with different PR. The traffic density affects the MRR when regular forwarding is used. IRR is the product of the MRR and PR, so the percentage of equipped vehicles has a strong influence on the IRR, as the upper limit of the IRR (when MRR $=100 \%$ ) is the PR.

\section{A. Simulation Setup}

We use VANET modules [15] that we developed for $n s-3$ [16] to examine DTMon's ability to collect high-quality traffic data, especially travel time and SMS, in transient flow traffic (i.e., non-recurring congestion). We have implemented and use Treiber's Intelligent Driver Model (IDM) [17] and MOBIL lane changing which are well-known car-following models. We use the $n s-3$ default log-distance fading model for the distribution of the attenuation of the signal in our network.

As shown in Figure 3, $\mathrm{TO}_{1}$ is located $1 \mathrm{~km}$ away from the entrance of a bi-directional four-lane highway. $\mathrm{TO}_{5}$ is located 5 $\mathrm{km}$ away from the beginning of the highway as an optional secondary TO for the cases when RF+SAC is used. The vehicles enter the highway with a medium flow rate (average $1800 \mathrm{veh} / \mathrm{h}$ ) to simulate slightly sparse traffic where the possibility of message reception by TOs is much lower than in dense traffic [1]. Trucks, which keep larger inter-vehicle spacing and have slower acceleration and deceleration than cars, comprise $20 \%$ of vehicles. The desired speed of all vehicles is $65 \pm 5 \mathrm{mph}(29 \pm 2.2 \mathrm{~m} / \mathrm{s})$.

We induce congestion by stopping a vehicle in the first lane $1.5 \mathrm{~km}$ away from the entrance, between $\mathrm{VS}_{1}$ and $\mathrm{VS}_{2}, 5$ minutes after the simulation has started. The stopped vehicle is outside the communication range $(300 \mathrm{~m})$ of $\mathrm{TO}_{1}$ and is relatively far from $\mathrm{TO}_{5}$. The vehicle will remain stopped for 5 minutes, then the vehicle will start moving, allowing traffic flow to gradually return to normal. During the stopping phase, following vehicles will slow and try to change lanes around the stopped vehicle. This causes traffic congestion in both lanes. We have observed through experimentation that 5 minutes is long enough to create a nonrandom transient flow. Note that congestion could have occurred at any point on the road and 
virtual strips can be defined dynamically by TOs. We named only those VS that surround the starting point of the congestion.

The performance of DTMon to collect traffic data is compared with the actual simulation status (ground truth) at $\mathrm{VS}_{2}(2 \mathrm{~km}$ away from the highway entrance and $0.5 \mathrm{~km}$ from the stopped vehicle). To compare our system with AVL, we assume that some percentage of the trucks are able to periodically communicate directly to an operating center to report their current status. For comparison with fixed point sensors and detectors (e.g., ILDs or microwave radar sensors), we use actual simulation data sampled from $\mathrm{VS}_{1}$ and $\mathrm{VS}_{2}$. We execute 10 runs of the simulation $(20 \mathrm{~min}$ each) for each experiment. We test PRs of 5, 10, 25, 50, and $100 \%$.

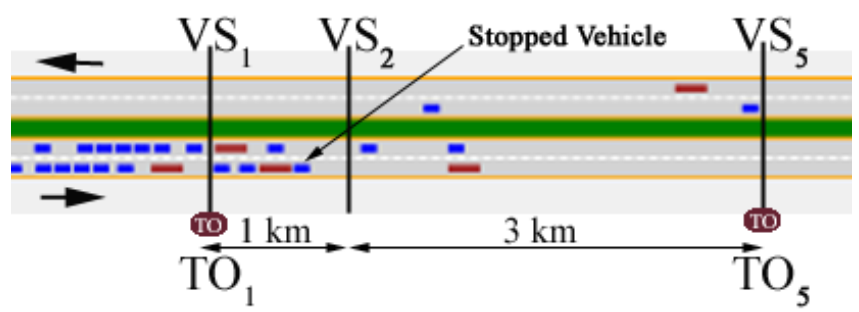

Figure 3. Location of TOs and virtual strips (not to scale).

\section{B. MRR and IRR using RF or RF+SAC}

For strips within transmission range of $\mathrm{TO}_{1}\left(\right.$ e.g., $\left.\mathrm{VS}_{1}\right)$, the message reception rate (MRR) is $100 \%$ and the information reception rate (IRR) equals the market penetration rate (PR). For farther strips where message delivery is required, the MRR using regular forwarding (RF) varies according to the distance from the TO and the PR. When RF+SAC is used, only PR affects the MRR.

Table I shows the MRR for RF, RF using traffic in the opposite direction, and RF+SAC for different penetration rates. With low PR, no messages were able to be forwarded using RF. Even using traffic in the opposite direction does not improve the MRR. In $25 \% \mathrm{PR}, \mathrm{TO}_{1}$ receives only $5.71 \%$ of messages (only $1.43 \%$ of total information) from $\mathrm{VS}_{2}$. Only $\mathrm{RF}+\mathrm{SAC}$ was able to deliver messages with full MRR. In medium PR (e.g., around 50\%), RF can deliver almost half of the messages although the IRR still remains below $30 \%$. Using traffic in opposite direction only improved MRR by $7 \%$. In $100 \% \mathrm{PR}, \mathrm{TO}_{1}$ misses about $20 \%$ of messages when RF alone is used (due to medium traffic flow rate and high inter-vehicle spacing). Using traffic in opposite direction for delivery improves the MRR by $15 \%$.

TABLE I. MRR FOR DIFFERENT PENETRATION RATES.

\begin{tabular}{|c|c|c|c|c|}
\hline PR & Actual & $\mathbf{R F}$ & $\mathbf{R F}+\mathbf{w} / \mathbf{p p}$ & $\mathbf{R F + S A C}$ \\
\hline $5 \%$ & 100 & 0.00 & 0.00 & 100 \\
\hline $25 \%$ & 100 & 5.71 & 5.71 & 100 \\
\hline $50 \%$ & 100 & 52.03 & 56.20 & 100 \\
\hline $100 \%$ & 100 & 79.50 & 91.01 & 100 \\
\hline
\end{tabular}

Table II shows the corresponding IRR. Note that, having low IRR in low penetration rates means that the system cannot estimate traffic flow rate or density. For example, DTMon in $25 \%$ PR can only count $1.43 \%$ (25\% with SAC) of vehicles in the traffic, thus the flow rates estimated by DTMon will be 0.014 ( 0.25 with SAC) of the actual flow rate on the road. This is a limitation of any system that does not directly count the physical presence of vehicles.

TABLE II. IRR FOR DIFFERENT PENETRATION RATES.

\begin{tabular}{|c|c|c|c|c|}
\hline PR & Actual & $\mathbf{R F}$ & $\mathbf{R F}+$ w/opp & $\mathbf{R F + S A C}$ \\
\hline $5 \%$ & 100 & 0.00 & 0.00 & 5 \\
\hline $25 \%$ & 100 & 1.43 & 1.43 & 25 \\
\hline $50 \%$ & 100 & 26.01 & 28.10 & 50 \\
\hline $100 \%$ & 100 & 79.50 & 91.01 & 100 \\
\hline
\end{tabular}

Figure 4 shows the percentage of messages that were forwarded or carried using $\mathrm{RF}+\mathrm{SAC}$ in different penetration rates. Even in $100 \%$ PR, $20 \%$ of messages were carried. Figure 4 also shows that with $100 \%$ PR using RF, in which no messages are carried, DTMon will count $20 \%$ fewer vehicles than actually exist. This is because inter-vehicle spacing can still be above the DSRC transmission range $(300 \mathrm{~m})$ in medium traffic flow. This is especially true for sections after a point of congestion but before $\mathrm{VS}_{2}$ where the traffic flow rate decreases and the gap among vehicles increases.

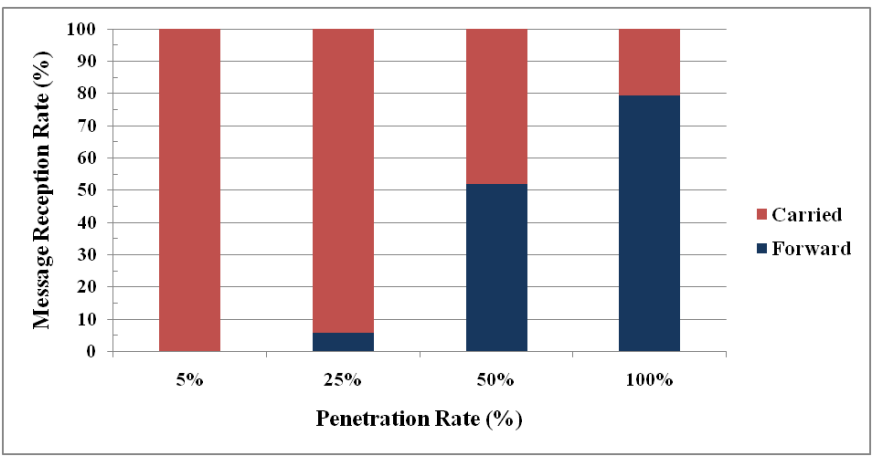

Figure 4. MRR from $\mathrm{VS}_{2}$ using RF+SAC in different PRs.

\section{Travel Time and Space Mean Speed}

First, we show that ILDs cannot accurately estimate travel times during transient traffic flow.

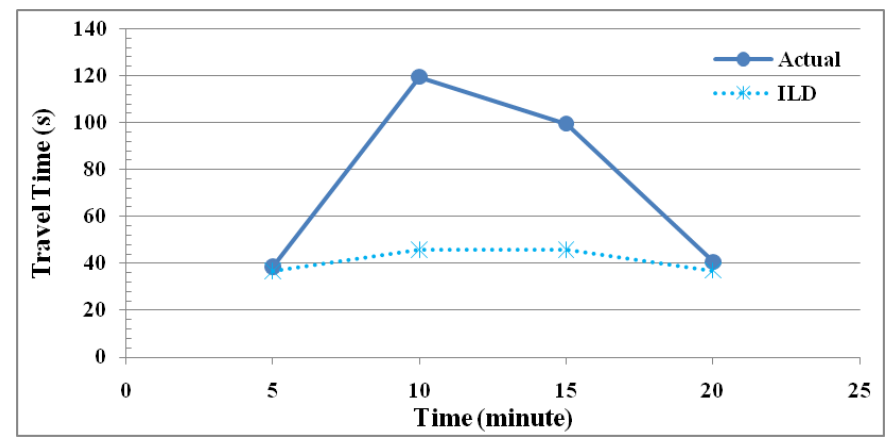

Figure 5. Estimated travel time by ILDs compared to actual (aggregation every 5 minutes).

Figure 5 shows the travel time in seconds, averaged every 5 minutes, for ILDs along with the actual (ground truth) travel times. Recall that congestion starts from beginning of the second interval ( $t=5$ minutes) and reaches its peak at the end of this interval ( $t=10$ minutes) when it starts to release during 
the third interval ( $t=10-15$ minutes). Since the ILDs estimate the travel time as the segment size divided by the averaged traffic spot speed, they cannot accurately follow the transient nature of the non-recurring congestion event.

Probe-based monitoring can perform better than ILDs for measuring travel times and SMS, so we compare the estimated travel time and SMS during each interval $(1 \mathrm{~min}$ or $5 \mathrm{~min}$ ) among data collected using RF, RF+SAC, and AVL. RF $\mathrm{p}_{\mathrm{p}}$ is RF when $\mathrm{PR}$ is $p$. In AVL where equipped vehicles are only trucks, $\mathrm{AVL}_{\mathrm{p}}$ is AVL when $p$ percentage of the total trucks in traffic are equipped. For example, $\mathrm{AVL}_{25}$ means $25 \%$ of the trucks in traffic belong to AVL and are equipped. $\mathrm{AVL}_{25}$ can be compared with $\mathrm{RF}_{5}$ in DTMon since $5 \%$ of the total population of vehicles are equipped (20\% of all vehicles are trucks).

Tables III and IV show the results of a t-test with alpha 0.05 (95\% confidence) for travel time (in seconds) in four 5-minute intervals, as well as the average travel time and SMS (in $\mathrm{m} / \mathrm{s}$ ) for the entire 20 minutes with $5 \% \mathrm{PR}$. The $t$-Stat column is the result of the t-test, showing if the mean of the samples is larger or smaller than the mean of the actual data. The $p$-Value column is the probability that the sample and the actual data come from different distributions. If $p$-Value is less than alpha (0.05), then the sample population and the actual population are deemed to have a significant statistical difference.

TABLE III. T-TEST AVL 25 AND ACTUAL.

\begin{tabular}{|c|c|c|c|c|c|c|c|}
\hline \multirow{2}{*}{$\begin{array}{c}\text { Time } \\
(\mathbf{m i n})\end{array}$} & \multicolumn{2}{|c|}{ Actual } & \multicolumn{2}{|c|}{ AVL $_{\mathbf{2 5}}$} & \multirow{2}{*}{ t-Stat } & \multirow{2}{*}{ p-Value } & \multirow{2}{*}{ Sig.? } \\
\hline $0-5$ & 38.55 & 0.40 & 43.12 & 0.03 & -1.5326 & 0.0393 & Yes \\
\hline $5-10$ & 119.51 & 0.46 & 138.01 & 0.02 & -7.0277 & 0.0055 & Yes \\
\hline $10-15$ & 99.59 & 0.32 & 127.86 & 0.60 & -1.8161 & 0.0018 & Yes \\
\hline $15-20$ & 40.62 & 0.28 & 42.97 & 1.10 & -2.1121 & 0.0400 & Yes \\
\hline $0-20$ & 74.57 & 1456.39 & 87.99 & 1163.09 & -0.8172 & 0.0360 & Yes \\
\hline SMS & 13.41 & - & 11.36 & - & - & - & Yes \\
\hline
\end{tabular}

TABLE IV. T-TEST RF 5 +SAC AND ACTUAL.

\begin{tabular}{|c|c|c|c|c|c|c|c|}
\hline \multirow{2}{*}{$\begin{array}{l}\text { Time } \\
(\min )\end{array}$} & \multicolumn{2}{|c|}{ Actual } & \multicolumn{2}{|c|}{$\mathrm{RF}_{5}+\mathrm{SAC}$} & \multirow{2}{*}{ t-Stat } & \multirow{2}{*}{ p-Value } & \multirow{2}{*}{ Sig.? } \\
\hline & Mean & Var & Mean & Var & & & \\
\hline $0-5$ & & & & & & & No \\
\hline & & & & & & & No \\
\hline & & & & & & & Yes \\
\hline $15-20$ & 40.62 & 0.28 & 39.38 & 21.68 & & & No \\
\hline $0-20$ & 74.57 & 1456.39 & 69.77 & 1025.95 & 0.0773 & 0.9391 & No \\
\hline SMS & 13.41 & - & 14.33 & - & - & 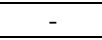 & No \\
\hline
\end{tabular}

Travel time and SMS estimated by $\mathrm{AVL}_{25}$ systems have significant difference compared to the actual data as shown in Table III. Even in $5 \% \mathrm{PR}, \mathrm{RF}_{5}+\mathrm{SAC}$ provides travel times and SMS with no significant difference from the actual travel time and SMS as shown in Table IV. In addition, the eminent change in travel time with a large mean difference occurring in the second and third interval shows the possibility of congestion or transition in traffic flow.

Figure 6 shows the travel times for actual, $\mathrm{AVL}_{25}$, and $\mathrm{RF}+\mathrm{SAC}_{5}$. Note that $\mathrm{RF}_{5}$ has $0.0 \mathrm{MRR}$ and therefore no travel time can be estimated. As indicated in Tables III and IV, $\mathrm{RF}+\mathrm{SAC}_{5}$ can produce travel times much closer to the actual traffic status than $\mathrm{AVL}_{25}$.

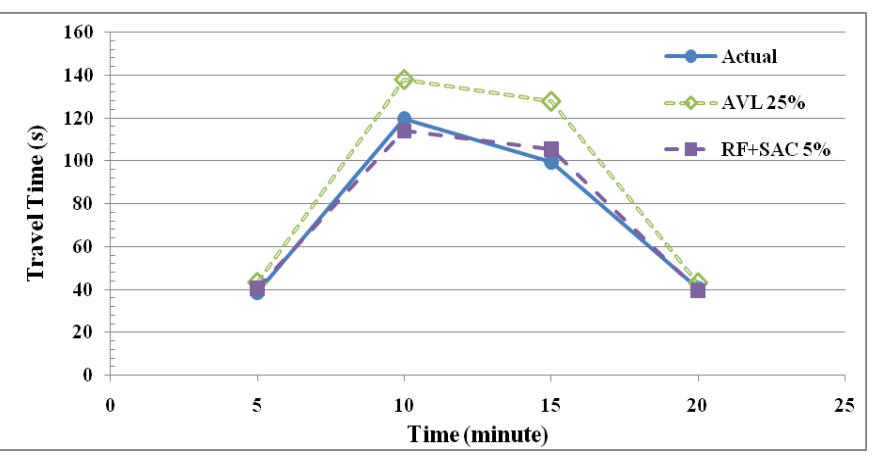

Figure 6. Average travel time (aggregation every 5 minutes).

Table $\mathrm{V}$ shows that even $\mathrm{AVL}_{100}$ provides significantly different estimation of travel time and SMS compared with the actual status of the highway. Table VI shows that $\mathrm{RF}_{50}$ (and by extension, $\mathrm{RF}+\mathrm{SAC}_{50}$ ) can provide high quality estimation of travel time and SMS that is not significantly different than the actual traffic conditions. The addition of SAC to RF will only add to the data quality because even more data can be delivered than with RF alone. Table VII shows the comparison between $\mathrm{RF}_{50}$ and $\mathrm{RF}+\mathrm{SAC}_{50}$. Some of the messages with $\mathrm{RF}+\mathrm{SAC}$ may have higher message delay (which we will discuss in Section VI.D), but as long as the delay is less than the aggregation interval, the data can be used in the traffic analysis.

TABLE V. T-TEST AVL $\mathrm{AL}_{100}$ AND ACTUAL.

\begin{tabular}{|c|c|c|c|c|c|c|c|}
\hline \multirow{2}{*}{$\begin{array}{c}\text { Time } \\
(\mathbf{m i n})\end{array}$} & \multicolumn{2}{|c|}{ Actual } & \multicolumn{2}{c|}{ AVL $_{\mathbf{1 0 0}}$} & \multirow{2}{*}{ t-Stat } & \multirow{2}{*}{ p-Value } & \multirow{2}{*}{ Sig.? } \\
\hline $0-5$ & 38.55 & 0.40 & 44.95 & 0.01 & -15.8148 & 0.0402 & Yes \\
\hline $5-10$ & 119.51 & 0.46 & 144.98 & 0.0007 & -54.8502 & 0.0116 & Yes \\
\hline $10-15$ & 99.59 & 0.32 & 139.86 & 0.03 & -153.0912 & 0.0041 & Yes \\
\hline $15-20$ & 40.62 & 0.28 & 44.79 & 0.08 & -23.6415 & 0.0269 & Yes \\
\hline $0-20$ & 74.57 & 1456.39 & 93.64 & 2722.33 & -3.4170 & 0.0111 & Yes \\
\hline SMS & 13.41 & - & 10.67 & - & - & - & Yes \\
\hline
\end{tabular}

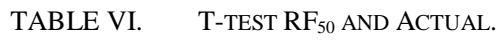

\begin{tabular}{|c|c|c|c|c|c|c|c|}
\hline \multirow{2}{*}{$\begin{array}{l}\text { Time } \\
(\mathbf{m i n})\end{array}$} & \multicolumn{2}{|c|}{ Actual } & \multicolumn{2}{|c|}{$\mathbf{R F}_{50}$} & \multirow{2}{*}{ t-Stat } & \multirow{2}{*}{ p-Value } & \multirow{2}{*}{ Sig.? } \\
\hline & Mean & Var & Mean & Var & & & \\
\hline $0-5$ & 38.55 & & & & & & \\
\hline 5- & 119. & & & & & & No \\
\hline & 99. & & & 367. & & & No \\
\hline $15-20$ & 40.62 & 0.28 & 39.17 & 1.35 & 3.2641 & & No \\
\hline $0-20$ & 74.57 & 1456.39 & & 1918.00 & -0.963 & & No \\
\hline SMS & 13.41 & & 12.59 & & & . & No \\
\hline
\end{tabular}

TABLE VII. T-TEST $\mathrm{RF}_{50}+\mathrm{SAC}$ AND $\mathrm{RF}_{50}$.

\begin{tabular}{|c|c|c|c|c|c|c|c|}
\hline \multirow{2}{*}{$\begin{array}{c}\text { Time } \\
(\mathbf{m i n})\end{array}$} & \multicolumn{2}{|c|}{$\mathbf{R F}_{\mathbf{5 0}}$} & \multicolumn{2}{c|}{$\mathbf{R F}_{\mathbf{5 0}}+\mathbf{S A C}$} & \multirow{2}{*}{-Stat } & \multirow{2}{*}{ p-Value } & \multirow{2}{*}{ Sig.? } \\
\cline { 2 - 6 } & Mean & Var & Mean & Var & & & \\
\hline $5-5$ & 39.00 & 2.01 & 38.94 & 0.01 & 0.0521 & 0.9668 & No \\
\hline $5-10$ & 115.78 & 9.85 & 116.72 & 0.15 & -0.4858 & 0.7120 & No \\
\hline $10-15$ & 123.55 & 367.38 & 97.92 & 0.01 & 1.8805 & 0.3111 & No \\
\hline $15-20$ & 39.17 & 1.35 & 38.84 & 0.05 & 0.5075 & 0.7010 & No \\
\hline $0-20$ & 79.37 & 1918.00 & 73.10 & 1388.43 & 1.2612 & 0.2476 & No \\
\hline SMS & 12.59 & - & 13.67 & - & - & - & No \\
\hline
\end{tabular}

Figures 7 and 8 show the estimated travel time and SMS, respectively, in $50 \% \mathrm{PR}$. Because of the $100 \% \mathrm{MRR}$ that $\mathrm{RF}+\mathrm{SAC}$ provides, DTMon is able to track the actual traffic status even during the transient flow periods. 


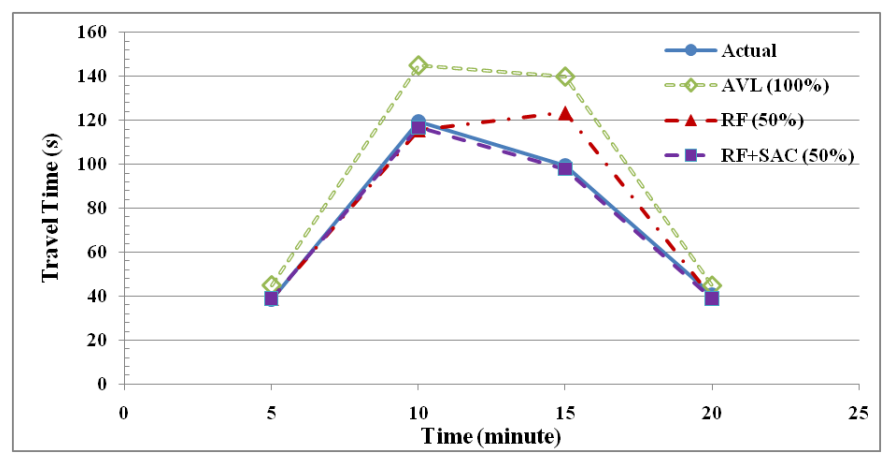

Figure 7. Travel time in 50\% PR (aggregation every 5 minutes).

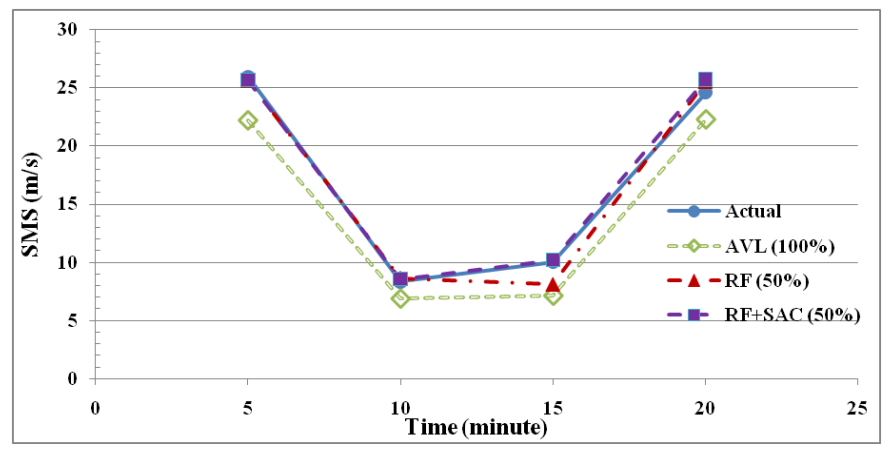

Figure 8. SMS in 50\% PR (aggregation every 5 minutes).

Figure 9 shows the trend in SMS when the aggregation interval is 1 minute. This indicates that transitions in traffic flow rate can affect travel times and speeds. Even at this level of aggregation, $\mathrm{RF}+\mathrm{SAC}$ can track the actual status closely.

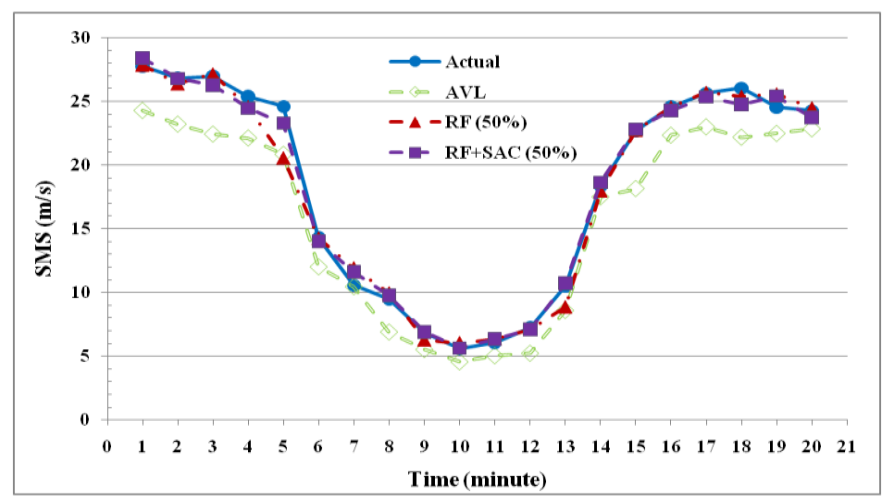

Figure 9. SMS in 50\% PR (aggregation every minute).

Table VIII summarizes the comparison of DTMon with conventional sensors and detectors currently in use as well as other probe vehicle-based systems, such as AVL. Note that WLT system performance is very similar to AVL except that WLT has also issues with accurately detecting the location and timing of vehicles at a specific point.

TABLE VIII. COMPARISON OF DTMON WITH OTHER TECHNOLOGIES.

\begin{tabular}{|c|c|c|c|}
\hline Good Estimate? & $\begin{array}{c}\text { Sensors and } \\
\text { Detectors }\end{array}$ & AVL & DTMon \\
\hline Travel Time & Not Available & Overestimate & Yes \\
\hline SMS & Not Available & Underestimate & Yes \\
\hline
\end{tabular}

As mentioned earlier, point-based sensors and detectors cannot directly measure travel times. AVL systems are typically installed in commercial trucks, which have different characteristics than the majority of traffic (slower acceleration/deceleration, larger inter-vehicle gaps, resulting in longer travel times).

\section{Latency (Message Delay)}

Sensors and detectors sense vehicles instantly. Their collected data are usually transferred to a TMC periodically via cable or wireless communication with some amount of latency. The aggregation usually happens every 5 minutes $[5,6]$. In AVL systems, vehicles' location and speed are probed periodically with negligible delay but these locations and speeds must be interpolated for a desired location and specific point on the road. For example, a vehicle with maximum speed $30 \mathrm{~m} / \mathrm{s}$ can be probed anywhere around $\mathrm{VS}_{2}$ in range of 15 meters when sampling period is every 5 seconds (which is a high rate). In the best case, a vehicle's location is probed when it is exactly passing $\mathrm{VS}_{2}$ at probe time. With a lower sampling rate, this offset increases.

Latency in DTMon varies depending upon several factors. As described in Section V.A, the message delay of a message is the time difference between when the message was generated by the vehicle and when it is received by a TO. The variation in the delay depends on the method of message delivery. For example, RF adds very small delay (in terms of milliseconds) while store-and-carry adds some travel time to the carried messages. In the previous section, we showed that TOs are capable of collecting and estimate high quality travel times and speeds as well as their trends even in traffic with transient flow rate and with various market penetration rates.

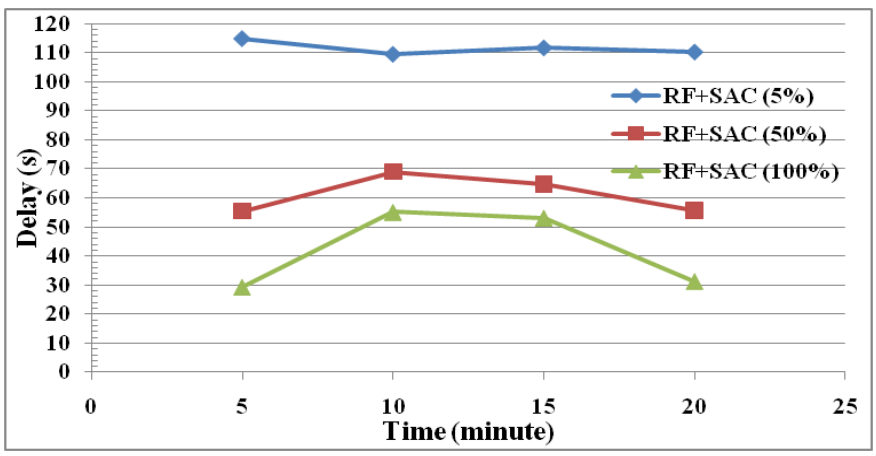

Figure 10. Message delay.

Figure 10 shows the message delay when $\mathrm{RF}+\mathrm{SAC}$ is used with different PR. In 5\% PR, the MRR (of messages sent from $\mathrm{VS}_{2}$ to $\mathrm{TO}_{1}$ ) is zero when RF is used and only RF+SAC could deliver the messages. This increases the message delay by the average travel time from $\mathrm{VS}_{2}$ to $\mathrm{TO}_{5}$. In medium PR like 50\%, almost half of the messages could be forwarded to $\mathrm{TO}_{1}$, with an average message delay of $7 \mathrm{~ms}$. The rest of the messages are carried to $\mathrm{TO}_{5}$. Congestion during the second and third interval (between 5-10 minutes) also adds additional delay to the messages that are carried. In high PR like 100\%, more messages are forwarded (80\%) than carried $(20 \%)$ and the average message delay becomes lower.

Message delay can be a factor in selecting the method of message delivery. In Tables VI and VII we showed that in 50\% $\mathrm{PR}$ and higher, RF and RF+SAC can provide high quality 
estimation of traffic speed and travel time and can also sense transition and trends in traffic flow based on travel time and SMS with $95 \%$ confidence. The advantage of using RF alone is that we can use a single TO instead of multiple TOs to cover the area with latency below one second. The disadvantage of $\mathrm{RF}$ is a lower IRR especially for VS far from a TO. In contrast, $\mathrm{RF}+\mathrm{SAC}$ results in higher IRR, especially in low PR, but with added latency. This latency can vary according to the traffic conditions and the distance from TOs.

Message delay can also be used to show how fresh the generated reports are. This may be crucial in real-time traffic monitoring. For example, aggregation may happen every minute as shown in Figure 9, but during congestion the message delay was 70 seconds for $\mathrm{RF}+\mathrm{SAC}_{50}$ (Figure 10), resulting in deviations from the actual traffic status.

\section{E. Count Information}

Count-based metrics such as flow rate, volume, and density can only be estimated when the market penetration is high. Figure 11 shows the computed flow rate by DTMon during each time interval. Only $20 \%$ of traffic are trucks, therefore the estimated flow rate using AVL is inaccurate. $\mathrm{RF}_{100}$ on average has $79.50 \%$ IRR and cannot count $21.5 \%$ of vehicles that passed $\mathrm{VS}_{2} . \mathrm{RF}_{100}+\mathrm{SAC}$ collects all messages and reports the same flow rate as ILDs and the actual traffic status. In $100 \%$ $\mathrm{PR}, \mathrm{RF}$ and $\mathrm{RF}+\mathrm{SAC}$ can detect the transition in flow rate as shown in Figure 11. Thus, high PR scenarios, DTMon can be a good replacement system for sensor and detectors. In low or medium PR, DTMon can be used to augment current systems and add important travel time and SMS data.

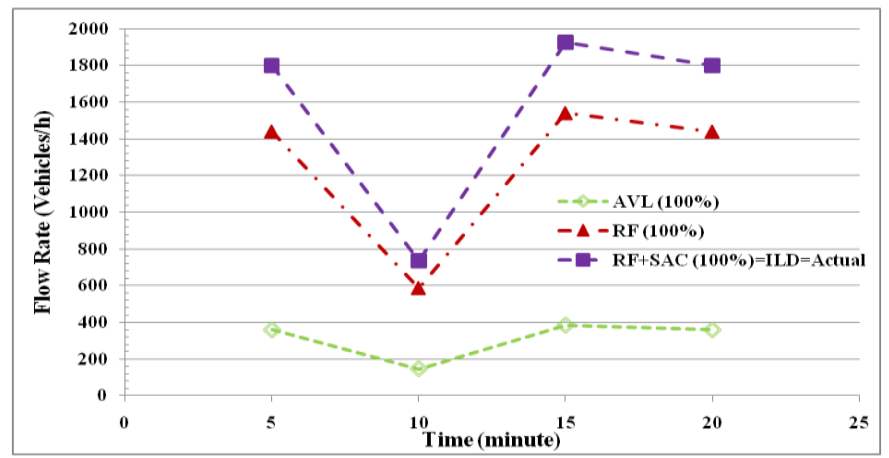

Figure 11. Flow rate at $\mathrm{VS}_{2}$ in $100 \%$ PR (aggregation every 5 minutes).

\section{CONCLUSION AND FUTURE WORK}

In this paper, we showed that DTMon can provide high quality travel time and SMS data in transient flow traffic, such as that caused by non-recurring congestion. Currently-deployed sensors and detectors can only estimate these metrics, and with transient flow traffic, the estimates are often far off from reality. With low market penetration rates, DTMon's measurements may incur some delay caused by vehicles having to carry their data to a nearby TO. But, even in these situations, DTMon can be used to augment current fixed point sensors and detectors. With high market penetration rates, DTMon could replace these sensors and detectors altogether. In addition to providing up-to-date travel statistics, DTMon can be used to detect transitions in traffic flow using travel time and speed measurements gathered from dynamically-defined virtual strips. We have shown that DTMon provides better performance than AVL systems in monitoring these metrics. The decision regarding which of DTMon's message delivery methods relies on the market penetration rate, message delay, and acceptable latency.

In our future work, we will further investigate the use of dynamically-defined virtual strips in DTMon to find and track the end-of-queue in transient traffic flows caused by congestion.

\section{ACKNOWLEDGMENTS}

This work was supported in part by the National Science Foundation under grant CNS-0721586.

\section{REFERENCES}

[1] H. Arbabi, M. C. Weigle, "Monitoring Free Flow Traffic using Vehicular Networks", In Proc. of the IEEE Intelligent Vehicular Communications System Workshop (IVCS). Jan 2011, to appear.

[2] H. Arbabi, M. C. Weigle, "Using Vehicular Networks to Collect Common Traffic Data", In Proc. of the ACM International Workshop on Vehicular Internetworking (VANET). Beijing, Sept. 2009, pp. 117-118.

[3] US Department of Transportation. "Standard Specification for Telecommunications and Information Exchange Between Roadside and Vehicle Systems - $5 \mathrm{GHz}$ Band Dedicated Short Range Communications (DSRC) Medium Access Control (MAC) and Physical Layer (PHY) Specifications.", ASTM E2213-03, Aug 2003.

[4] M. Fontaine, "Traffic Monitoring" in Vehicular Networks from Theory to Practice, Ed. S. Olariu, M. C. Weigle, CRC Press, 2009.

[5] INRIX, available at http://inrix.com/.

[6] Mobile Century/Mobile Millennium project with Nokia available at http://traffic.berkeley.edu.

[7] PATH's GEMS project available at http://www.path.berkeley.edu.

[8] E. Mazloumi, et al., "Using GPS Data to Gain Insight into Public Transport Travel Time Variability," Journal of Transportation Engineering-Asce, vol. 136, pp. 623-631, Jul 2010.

[9] A. Faghri, K. Hamad, "Travel time, speed, and delay analysis using an integrated GIS/GPS system," Canadian Journal of Civil Engineering, vol. 29, pp. 325-328, Apr 2002.

[10] B. Hull, V. Bychkovsky, Y. Zhang, K. Chen, M. Goraczko, A. Miu, E. Shih, H. Balakrishnan, and S. Madden, "CarTel: a distributed mobile sensor computing system," In Proc. of the International Conference on Embedded Networked Sensor Systems (SenSys), 2006.

[11] T. Nadeem, S. Dashtinezhad, and C. Liao, "Traffic view: A Scalable Traffic Monitoring System," In Proc. of the IEEE Int'l Conf. on Mobile Data Management (MDM), 2004, pp.13-21.

[12] T. Kitani, T. Shinkawa, N. Shibata, K. Yasumoto, M. Ito, T. Higashino, "Efficient VANET-based Traffic Information Sharing using Buses on Regular Routes," In Proc. of the IEEE Vehicular Technology Conference (VTC), May 2008, pp. 3031-3036.

[13] V. Sethi, et al., "Arterial Incident Detection Using Fixed Detector and Probe Vehicle Data," Transportation Research Part C-Emerging Technologies, vol. 3, pp. 99-112, Apr 1995.

[14] M. W. Sermons and F. S. Koppelman, "Use of vehicle positioning data for arterial incident detection," Transportation Research Part CEmerging Technologies, vol. 4, pp. 87-96, Apr 1996.

[15] H. Arbabi, M. C. Weigle, "Highway Mobility and Vehicular Ad-Hoc Networks in ns-3," In Proc. of the Winter Simulation Conference. Baltimore, MD, Dec 2010.

[16] ns-3 network simulator. Available at http://www.nsnam.org

[17] M. Treiber, A. Hennecke, and D. Helbing, "Congested traffic states in empirical observations and microscopic simulations," Phys. Rev. E, Stat. Phys. Plasmas Fluids Relat. Interdiscip. Top., vol. 62, no. 2, pp. 18051824, Aug 2000. 